\title{
MÉTODOS DE AVALIAÇÃO DE IMPACTOS DO CICLO DE VIDA: UMA DISCUSSÃO PARA ADOÇÃO DE MÉTODOS NAS ESPECIFICIDADES BRASILEIRAS
}

\section{LIFE CYCLE IMPACT ASSESSMENT METHODS: A DISCUSSION OF METHODS ADOPTION IN BRAZILIAN SPECIFITIES}

\author{
Cassiano Moro Piekarski ${ }^{1}$; Leila Mendes da Luz ${ }^{2}$; Lidiana Zocche ${ }^{3}$; Antonio Carlos de Francisco ${ }^{4}$ \\ ${ }^{1}$ Universidade Tecnológica Federal do Paraná - UTFPR - Ponta Grossa - Brasil \\ cassianopiekarski@gmail.com \\ ${ }^{2}$ Universidade Tecnológica Federal do Paraná - UTFPR - Ponta Grossa - Brasil \\ leila.mendesdaluz@gmail.com \\ ${ }^{3}$ Universidade Tecnológica Federal do Paraná - UTFPR - Ponta Grossa - Brasil \\ lidianazocche@hotmail.com \\ ${ }^{4}$ Universidade Tecnológica Federal do Paraná - UTFPR - Ponta Grossa - Brasil \\ acfrancisco@utfpr.edu.br
}

\begin{abstract}
Resumo
A Avaliação do Impacto do Ciclo de Vida (AICV) é realizada mediante a associação dos dados do inventário do ciclo de vida (ICV) a categorias de impacto específicas. Este processo pode receber o auxilio de diferentes métodos de avaliação. Porém, os métodos existentes não condizem com as especificidades brasileiras, e isso representa uma das dificuldades encontradas na avaliação do ciclo de vida no Brasil. Deste modo este estudo tem como objetivo, discutir a adoção de múltiplos métodos para AICV nas especificidades brasileiras. Para isso, desenvolveu-se uma pesquisa de caráter exploratório, onde foram abordados conceitos referentes a ACV, métodos para AICV e a adoção de múltiplos métodos de avaliação com intuito de atender as especificidades brasileiras. Nota-se que a possibilidade de comparação dos resultados gerados por métodos diferentes apresenta-se como uma característica predominante, deste modo as incertezas geradas no estudo de ACV podem ser reduzidas adotando-se múltiplos métodos de avaliação.
\end{abstract}

Palavras-chave: Avaliação do Ciclo de Vida (ACV); Avaliação de Impactos do Ciclo de Vida (AICV); Métodos de AICV.

\section{Introdução}

A Análise do Ciclo de Vida (ACV) avalia os aspectos e impactos potencias associados a produtos, processos e serviços mediante a associação dos dados do inventário do ciclo de vida a categorias de impacto específicas, num processo que consiste na Avaliação do Impacto do Ciclo de Vida (AICV). 
Na AICV são consideradas várias categorias de impacto selecionadas de acordo com o objetivo e escopo definido no estudo. De acordo com CCI (2010), as emissões e recursos são atribuídos as categorias de impacto e convertidos em indicadores utilizando métodos de avaliação de impacto.

Estes métodos abrangem muitas categorias de impacto proporcionando diferentes fatores de caracterização (HAUSCHILD et al, 2012). Por isso, o desafio da AICV é avaliar o impacto potencial das substâncias emitidas utilizando um procedimento aplicável a todas as substâncias de forma consistente utilizando uma unidade comum de medida, e resultar em dados comparáveis entre categorias de impacto (PIZZOL et al, 2011a).

Para superar esses desafios, ao longo dos anos, os pesquisadores têm desenvolvido vários métodos para AICV (PIZZOL et al, 2011b). Porém, os métodos desenvolvidos consideram impactos ambientais globais e/ou relativos a regiões específicas onde foram desenvolvidos, como Canadá, Europa, Japão e Estados Unidos. Sendo assim, estes métodos não refletem necessariamente a situação de países como o Brasil, o qual ainda não possui métodos de AICV direcionados em específico para as características ambientais do país (SILVA, 2010).

Em decorrencia disso, uma das dificuldades encontradas na avaliação de impacto em estudos de ACV no Brasil é a falta de métodos que condizem com as especificidades brasileiras. Na tentativa de minimizar as incertezas geradas neste tipo de avaliação alguns estudos desenvolvidos, como Cavalett (2010), sugerem a adoção de mais de um método de AICV. Silva (2010) também ressalta que a utilização de mais de um método na avaliação de impactos é importante, pois permite verificar se os resultados obtidos variam conforme o método adotado, e quais as diferentes conclusões percebidas.

Diante disso, este estudo tem como objetivo discutir a adoção de múltiplos métodos para AICV nas especificidades brasileiras. Para isso, desenvolveu-se uma pesquisa básica de caráter exploratório, onde foram abordados conceitos referentes a ACV, métodos para AICV e a adoção de múltiplos métodos com intuito de atender as especificidades brasileiras. Nos tópicos a seguir são apresentados os principais pontos levantados na pesquisa sobre os assuntos em pauta e as considerações finas do estudo realizado.

\section{Avaliação do Ciclo de Vida - Visão Geral}

A ACV é uma ferramenta técnica de abordagem analítica e caráter gerencial que contribui para a avaliação dos aspectos ambientais e impactos potenciais associados a um produto ou atividade durante seu ciclo de vida (GARRAÍN, 2010; LOFGRE, TILLMAN, RINDE, 2011; CHAUHAN et al, 2011). 
Em termos gerais de acordo Lofgren, Tillman e Rinde (2011) o objetivo com relação ao uso da ACV é satisfazer os pedidos dos clientes e ao mesmo tempo conseguir um equilíbrio ideal entre o produto e o meio ambiente. A metodologia (ACV) desenvolveu e amadureceu durante as últimas décadas. As atividades atuais sobre bancos de dados, garantia de qualidade, consistência e harmonização dos métodos contribuíram para isso.

A atual metodologia regente para a ACV é normatizada pela ISO 14040 (ABNT, 2009). A metodologia engloba quatro diferentes fases: definição de objetivo e escopo, análise de inventário, avaliação de impacto e interpretação. A iteração entre estas fases pode ser observada na Figura 1.

Figura 1: Fases da avaliação do ciclo de vida

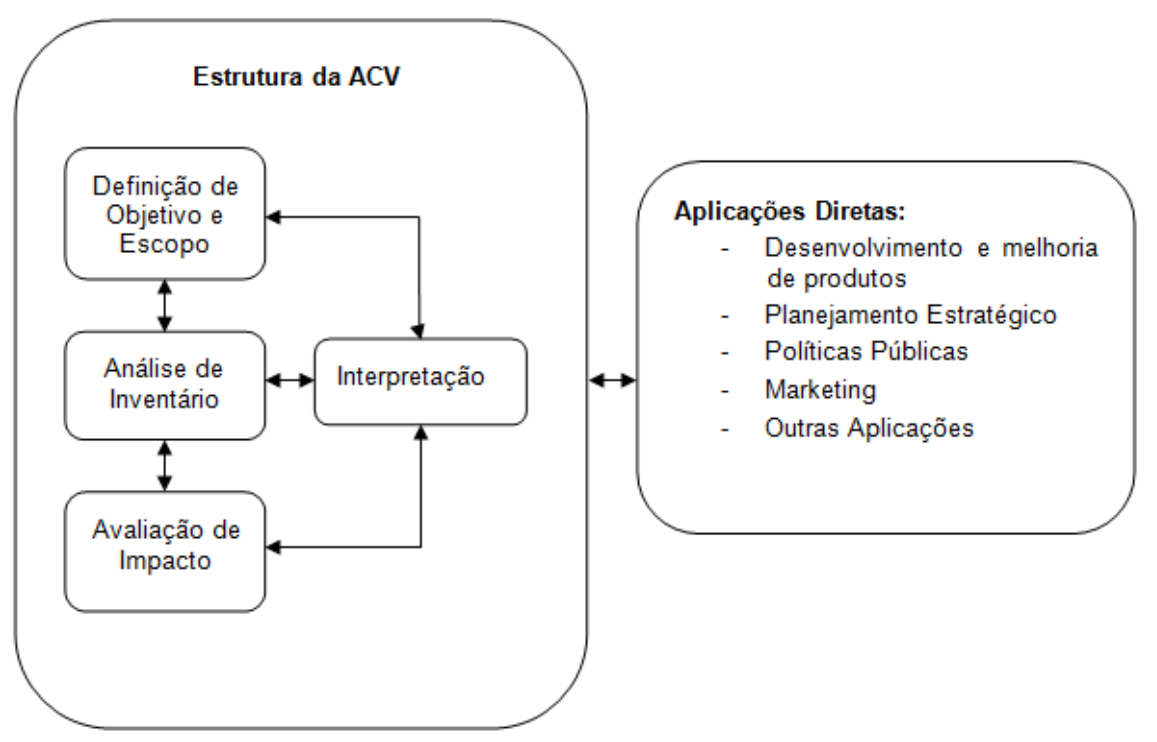

Fonte: ISO 14040 (ABNT, 2009)

A metodologia de aplicação de um estudo de ACV pode ser entendida como o conjunto de procedimentos necessários para que o estudo atinja aos objetivos propostos. As fases metodológicas da ACV podem ser descritas individualmente nos quatro subtópicos a seguir.

\subsection{Definição de Objetivo e Escopo}

A primeira fase da ACV compreende a definição do objetivo e escopo. O objetivo de um estudo ACV Deve declarar inequivocamente a aplicação pretendida, as razões para conduzir o estudo e o público-alvo (ABNT, 2009a).

Já o escopo compreende a abrangência do estudo. De acordo com os autores, Sherwani, Usmani, Varun (2010); Pieragostini, Mussat, Aguirre (2012); Zhou, Chang, Fane (2011), devem ser demonstrados claramente no escopo o sistema de produto a ser estudado, as fronteiras do sistema, a unidade funcional e os limites do sistema, conforme apresentados a seguir:

- Sistema de produto: a função do sistema é a finalidade de uso do produto; 
- Fronteiras do sistema: Interface entre um sistema de produto e o meio ambiente ou outros sistemas de produto;

- Unidade funcional: define explicitamente o serviço ou uma função fornecida pelo sistema de produtos, seu desempenho quantificado uso como uma unidade de referência num estudo de ACV, permitindo uma análise e comparação entre diferentes sistemas;

- Limites do sistema: estabelecem o que será considerado no âmbito do estudo e o que será excluído.

Além destes itens, outros elementos que compõe o escopo devem ser considerados se julgados necessários para garantir a qualidade do estudo.

Depois de definido os objetivos e escopo do estudo a próxima fase da ACV consiste na análise do inventario do ciclo de vida.

\subsection{Análise de Inventário}

A definição do objetivo e escopo de um estudo provê o plano inicial para a condução da fase de inventário do ciclo de vida de uma ACV. Esta fase envolve um extenso banco de dados de materiais envolvidos no produto ou sistema incluindo o levantamento, a coleta e a análise dos dados necessários para a ACV (JIJAKLI, 2012; OLSEN et al, 2001; GUINNÉ et al, 2011).

O inventário, como mensionado por Sherwani, Usmani e Varun (2010) deve, em princípio, conter dados sobre todas as entradas e saídas de cada processo individual do sistema examinado, como fluxos de poluentes, materiais e recursos (SUH, HUPPES, 2005; ZHOU, CHANG, FANE, 2011).

Esses dados são apresentados ao final desta fase de forma quantificada, que após o tratamento dos dados poderão então ser avaliados para obtenção dos impactos ambientais associados ao ciclo de vida do produto em questão através da próxima fase da ACV, a Avaliação de Impactos.

\subsection{Avaliação de Impactos do ciclo de vida}

A etapa de avaliação do impacto do ciclo de vida (AICV) consiste em estudar a significância dos impactos ambientais, a partir dos dados do inventário. Isso é feito por meio da análise dos potenciais impactos ambientais associados aos aspectos ambientais identificados na etapa de análise de inventário (JÚNIOR e DEMSJOROVIC, 2010).

A Norma ISO 14044 (ABNT, 2009b) indica que a esta fase consiste em elementos obrigatórios: seleção das categorias de impacto, classificação e caracterização e opcionais: a normalização, agrupamento, ponderação e análise adicional da qualidade dos dados. 
O primeiro elemento, etapa de seleção das categorias de impacto, identifica as preocupações ambientais, as categorias e os indicadores que o estudo utilizará. A seleção dessas categorias devem estar relacionadas ao sistema de produto em estudo, levando em consideração o objetivo e escopo do estudo.

A classificação correlaciona dados do inventário com as categorias de impacto ambiental. Deve-se declarar explicitamente qual categoria de impacto será levada em consideração. Nesta fase são as entradas e saídas do inventario que contribuem para causar impacto sobre o meio ambiente e são classificadas de acordo com o problema.

O terceiro e último elemento obrigatório da avaliação de impacto consiste na caracterização, nesta etapa de acordo com Myllyviita et al (2012), as contribuições para cada problema ambiental são quantificadas, os resultados dos indicadores (caracterização) envolvem a conversão dos resultados do inventário para unidades comuns e a agregação dos resultados convertidos dentro a mesma categoria de impacto.

Ao final da avaliação de impacto do ciclo de vida, tem-se como resultado final um perfil ambiental do sistema de produto em estudo, conforme definido no objetivo e escopo. Esses resultados serão interpretados na última fase da estrutura metodológica da avaliação do ciclo de vida.

\subsection{Interpretação}

A última fase da estrutura da avaliação do ciclo de vida compreende a interpretação dos resultados. Nesta fase todas as constatações da análise do inventário e avaliações do impacto são consideradas em conjunto (ABNT, 2009a). Os resultados a serem interpretados devem ser consistentes com o objetivo e escopo definidos no início do estudo. As interpretações devem levar a conclusões, explicitar limitações que podem tornar os objetivos iniciais inalcançáveis, identificar as principais fases do ciclo de vida que contribuem para os impactos ambientais e fornecer recomendações finais (LUZ, 2011; JJJAKLI et al, 2012; PIERAGOSTINI, MUSSAT, e AGUIRRE, 2012).

A fase de interpretação é considerada importante segundo Suer, Paledal e Norrman (2004), pois é a chave para tornar os resultados da avaliação de impacto comparável e compreensível.

Após a análise e interpretações dos resultados as conclusões obtidas permitirão a identificação de pontos críticos que necessitam de melhorias, inovação nos produtos ou processos de fabricação, visando à preservação ambiental.

\section{Métodos para AICV}


Para a realização da fase de AICV o cálculo dos indicadores de categorias de impacto, faz parte dos elementos obrigatórios apresentados pela norma ISO 14044. De acordo com Hauschild et al (2012), nesta fase do estudo, o impacto potencial de cada emissão de inventário e/ou fluxo de recursos para o meio ambiente é modelado quantitativamente de acordo com o mecanismo ambiental relevante utilizando um modelo de caracterização.

O modelo de caracterização calcula fatores de caracterização de substâncias específicas que expressam o impacto potencial de cada fluxo elementar em termos da unidade comum do indicador de categoria. Deste modo, os fatores de caracterização são multiplicados com os dados do inventário, e os resultados são os resultados da categoria de indicadores, expressos em uma unidade comum a todas as contribuições dentro da categoria de impacto (por exemplo, quilogramas equivalentes de $\mathrm{CO}_{2}$ de gases de efeito estufa que contribui para o impacto da categoria mudanças climáticas) (HAUSCHILD et al, 2012).

Estes modelos de caracterização são conhecidos como métodos para AICV. Apresentam características individuais específicas e podem ser classificados em duas categorias de acordo com sua abordagem: midpoint (ponto médio) e endpoint (ponto final).

De acordo com Cavalett et al (2012), ao nível midpoint todas as substâncias referentes ao ICV são adequadamente agregadas em categorias de impacto de acordo com uma característica comum na cadeia de causa efeito do mecanismo ambiental. Estas características não representam as consequências finais sobre o percurso ambiental das emissões listadas no inventário do ciclo de vida (ICV), mas são indicadores de impacto em potencial.

A modelagem endpoint consiste basicamente em caracterizar a gravidade ou as consequências de categorias de impacto de ponto médio nas áreas de proteção a nível de ponto final. Esta caracterização, a nível endpoint exige modelar todos os mecanismos ambientais que conectam os resultados do inventário, com o respectivo impacto sobre as áreas de proteção, sendo portanto, quantificadas as consequências (danos) finais das emissões (CAVALETT et al, 2012).

De acordo com Bare et al (2000) devido ao fato dos métodos endpoint serem direcionados ao dano, são geralmente considerados mais compreensíveis para os tomadores de decisão, apresentando mais relevância na tomada de decisão, no entanto, possuem alta subjetividade. Já, os métodos midpoint apresentam menor subjetividade, mas também possuem menor relevância no suporte à tomada de decisão.

As características mais relevantes num método de AICV são suas categorias de impacto ambiental (ALVARENGA, 2010). Sendo que cada método apresenta um conjunto de categorias específicas. Algumas das categorias de impacto comumente relacionados aos dados do ICV e a métodos midpoint e endpoint podem ser observadas na Figura 2. 
Figura 2 - Categorias de impacto a midpoint e endpoint

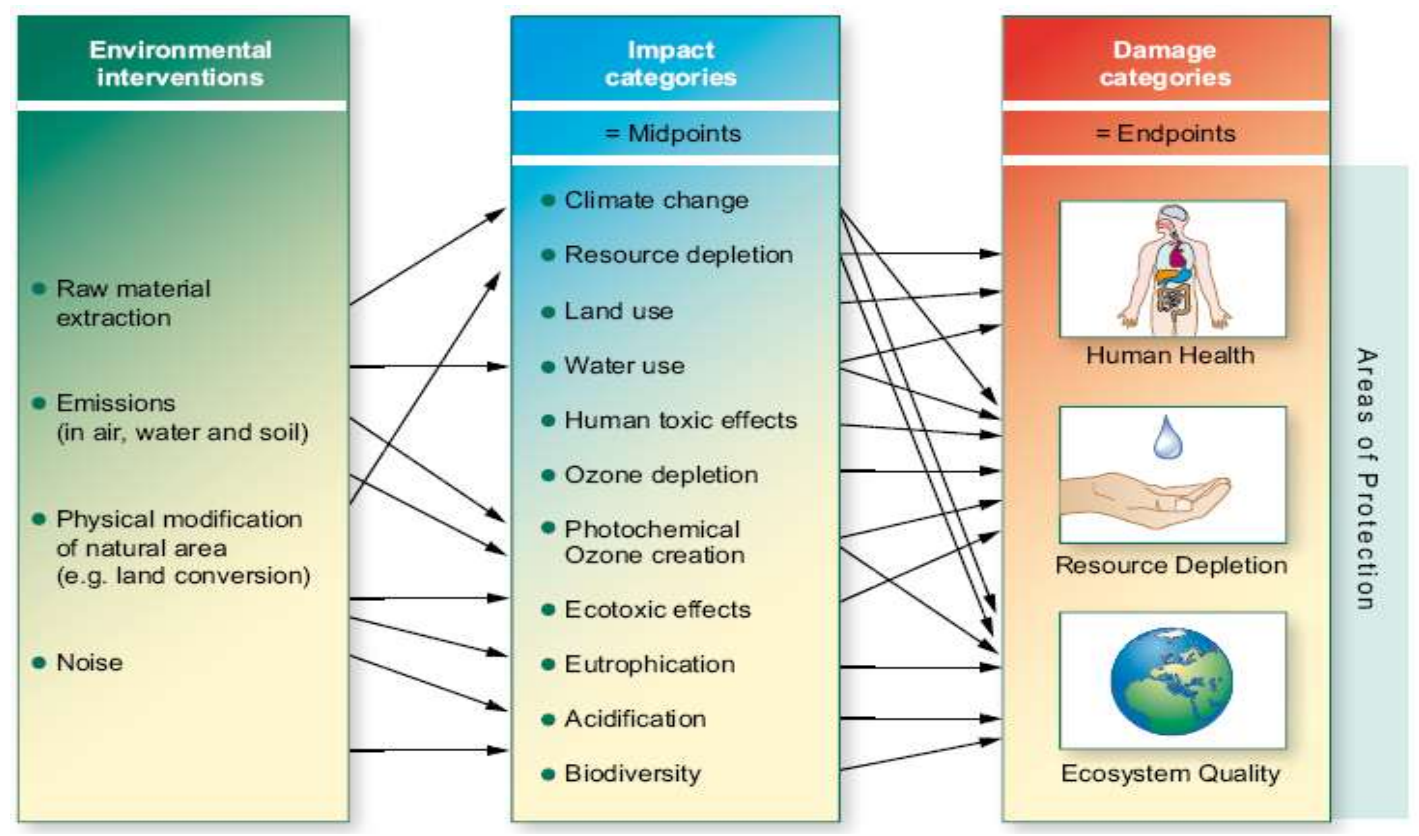

Fonte: United Nations Environment Programme (2011)

Vários métodos de avaliação de impacto têm sido desenvolvidos e aplicados em estudos de ACV, por exemplo: CML (SOUZA et al, 2012), Eco-indicador 99 (RESTREPO et al, 2012), EDIP 2003 (SANTOS, 2010), IMPACT 2002+ (SILVA, KAZIMI, HEJZLAR, 2010), ReCiPe (SILVA, 2012). Estes métodos estão entre os mais citados e aplicados (CCI, 2010) e serão discutidos com mais detalhes nos tópicos seguintes. Além destes, outros métodos também vem sendo aplicados, como os apresentados no Quadro 1.

Quadro 1 - Métodos para AICV

\begin{tabular}{|l|l|l|l|l|}
\hline \multicolumn{1}{|c|}{ Método } & \multicolumn{1}{|c|}{ País } & Abordagem & \multicolumn{1}{|c|}{ Finalidade do método } & \multicolumn{1}{c|}{$\begin{array}{c}\text { Detalhamento do } \\
\text { método }\end{array}$} \\
\hline USEtox & - & midpoint & $\begin{array}{l}\text { Fornecer fatores de caracterização para } \\
\text { toxicidade humana e ecotoxicidade de água } \\
\text { doce na AICV }\end{array}$ & $\begin{array}{l}\text { Hauschild et al (2008b); } \\
\text { Rosenbaum et al (2008) }\end{array}$ \\
\hline TRACI & $\begin{array}{l}\text { Estados } \\
\text { Unidos }\end{array}$ & midpoint & $\begin{array}{l}\text { Desenvolver um método de avaliação de } \\
\text { impacto que representa as condições nos } \\
\text { EUA, e que está em linha com a política de } \\
\text { EPA }\end{array}$ & Bare et al (2003) \\
\hline LUCAS & Canadá & midpoint & $\begin{array}{l}\text { Metodologia para AICV adaptada ao } \\
\text { contexto canadense }\end{array}$ & Bulle et al (2007) \\
\hline LIME & Japão & $\begin{array}{l}\text { Midpoint/ } \\
\text { endpoint }\end{array}$ & $\begin{array}{l}\text { Desenvolver listas de ponto médio } \\
\text { (caracterização), ponto final (avaliação de } \\
\text { danos) e a ponderação que reflete as } \\
\text { condições ambientais do Japão. }\end{array}$ & $\begin{array}{l}\text { Itsubo et al (2004); } \\
\text { Hayashi et al (2006) }\end{array}$ \\
\hline EPS & Suécia & endpoint & $\begin{array}{l}\text { Ajudar designers e desenvolvedores de } \\
\text { produtos em apoio à decisão. }\end{array}$ & $\begin{array}{l}\text { Steen (1999a); Steen } \\
\text { (1999b); Steen (2001) }\end{array}$ \\
\hline Ecopoints & Suíça & endpoint & Fornecer caracterização e factores de & Brand et al (1998); \\
\hline
\end{tabular}




\begin{tabular}{|l|l|l|l|l|}
\hline $\begin{array}{l}\text { (escassez } \\
\text { ecológica) }\end{array}$ & & $\begin{array}{l}\text { ponderação de várias emissões e extrações } \\
\text { com base em metas de políticas públicas e } \\
\text { objetivos. } \\
\text { O método original foi desenvolvido para } \\
\text { Suíça }\end{array}$ & $\begin{array}{l}\text { Frischknecht et al } \\
(2009)\end{array}$ \\
\hline MEEuP & - & midpoint & $\begin{array}{l}\text { Fornecer uma metodologia que permite } \\
\text { avaliar se, e em que medida vários produtos } \\
\text { que consomem energia (EuP) cumprem } \\
\text { determinados critérios que os tornam } \\
\text { elegíveis para implementação de medidas } \\
\text { sob a concepção ecológica dos produtos } \\
\text { consumidores de energia }\end{array}$ & Kemna et al (2005) \\
\hline $\begin{array}{l}\text { Pegada } \\
\text { ecológica }\end{array}$ & - & Endpoint & $\begin{array}{l}\text { Fornecer um indicador da área biológica } \\
\text { produtiva necessária para atender a demanda } \\
\text { humana. Abordagem composto. }\end{array}$ & $\begin{array}{l}\text { Wackernagel (2005); } \\
\text { Huijbregts et al (2006) }\end{array}$ \\
\hline
\end{tabular}

Fonte: Elaborado com base em CCI (2010) e ECOINVENT (2010).

Estes métodos estão integrados nos softwares de ACV normalmente utilizados como suporte para estudos de ACV (PIZZOL et al, 2011a). A escolha do método de avaliação é feita tomando-se como base a análise das questões ambientais relevantes para o ciclo de vida do produto estudado (SILVA, 2012). Pois, de acordo com Zhou et al (2011), não há informações claras ou até mesmo orientações para a escolha de métodos adequados para AICV.

\subsection{Eco-Indicator 99}

O Eco-indicator 99 foi desenvolvido na Holanda por um grupo científico especializado em estudos de ACV e ciências ambientais, com apoio do Ministério Holandês do Meio Ambiente. Este método é uma versão reestruturada do método Eco-Indicator 95, seu antecessor (GOEDKOOP et al, 2008).

$\mathrm{O}$ método de AICV Eco-Indicator possui uma abordagem orientada ao dano (endpoint). Ou seja, a cadeia de causa-efeito do ciclo de vida de um produto, processo ou serviço, é modelada até seus pontos finais, os danos. Estes são classificados em três classes de danos: Saúde Humana, Qualidade do Ecossistema e Recursos (GOEDKOOP; SPRIENSMA, 2000a).

Segundo Takeda (2008), a avaliação de impacto deste método consiste em duas partes: i) cálculo das mudanças no ambiente causadas pelo fluxo ambiental de um ciclo de vida e; ii) normalização e ponderação, a fim de estabelecer a seriedade destas mudanças (etapas opcionais da AICV).

Segundo Goedkoop; Spriensma (2000a), a estrutura metodológica do Eco-Indicator 99 envolve três fases: a caracterização dos efeitos ambientais; a avaliação dos danos ambientais sobre a saúde humana, a qualidade do ecossistema e recursos; e a normalização e a valoração dos impactos ambientais. Ao final é gerado um Eco-indicador ambiental expresso através de uma pontuação ponderada proveniente da soma das três classes de danos.

Segundo Alvarenga (2010), o método mais utilizado no grupo de métodos orientados ao 
dano é o Eco-Indicator 99. É recomendável que os usuários do mesmo consultem as publicações originais do método (GOEDKOOP et al 1998; GOEDKOOP \& SPRIENSMA 2000a; GOEDKOOP \& SPRIENSMA 2000b) para compreensão de detalhes e características de normalidade e ponderação do Eco-Indicator 99.

\subsection{2001}

O método CML 2001 (ou CML 01) surgiu por meio de estudos de um grupo de cientistas associados ao Centro de Ciências Ambientais da Universidade de Leiden, Holanda. O grupo publicou um guia operacional para as normas ISO (GUINÈE et al, 2001b; GUINÈE et al, 2001c), nomeado Dutch Handbook on LCA (CML). A proposta geral é fornecer orientações com guias operacionais para conduzir um estudo de ACV passo a passo.

O guia relata uma diferenciação na abordagem dos impactos ambientais. A abordagem orientada ao problema (midpoint) e a orientada ao dano (endpoint) foram diferenciadas. Neste aspecto, o método holandês CML 2001 foi criado com diferentes temas ambientais (categorias de impactos) relacionados à abordagem orientada ao problema (midpoint).

Segundo Takeda (2008), o CML "Guide" fornece uma lista de categorias de avaliação de impacto, que podem ser agrupadas em:

a) Categorias obrigatórias de impacto: São indicadores de categoria utilizados na grande maioria de ACV`s;

b) Categorias de impacto adicionais: Dizem respeito à existência de indicadores operacionais, porém não comumente inclusos em ACV`s;

c) Outras categorias de impacto: Quando não existem indicadores operacionais disponíveis, tornando-se impossível a inclusão quantitativa em estudos ACV.

As categorias de impacto obrigatórias estão disponíveis em diversos métodos de AICV, e permitem comparações de seus resultados entre diferentes métodos. As principais categorias de impacto do CML 2001 são: Acidificação, Depleção da camada de ozônio, Depleção de recursos abióticos, Ecotoxicidade, Eutrofização, Formação fotoquímica de ozônio troposférico, Mudança Climática, Oxidação fotoquímica, Radiação ionizante, Toxicidade humana, Uso do solo (GUINÉE, 2001a).

O método conta com fatores de caracterização específicos previamente quantificados para cada problema ambiental (midpoint) ou categoria de impacto. Neste sentido, as emissões relatadas na fase de ICV são convertidas em potencial de impacto ambiental através do produto entre sua respectiva quantidade e seu fator de caracterização na categoria de impacto conexa (GUINÉE, 2001a). 
A amplitude das categorias de impacto associadas ao CML 2001 releva sua importante representatividade na comunidade científica. Alvarenga (2010) afirma que o método mais utilizado em estudos ACV`s que adotam a abordagem midpoint é o CML 2001. Para os praticantes de ACV, é altamente recomendável que, quando usuários do CML 2001, consultem as referências originais (GUINÈE et al, 2001a; GUINÈE et al, 2001b; GUINÈE et al, 2001c) para o entendimento de detalhes específicos deste método de AICV.

\subsection{EDIP 2003}

A sigla EDIP refere-se à "Environmental Design of Industrial Products", em inglês. Em dinamarquês, o método é chamado UMIP. O método EDIP 2003 foi criado na Dinamarca e é uma evolução do EDIP 97, entretanto não o substitui. A versão de 2003 apresenta um modelo de caracterização diferenciado espacialmente, passando por uma abordagem que o coloca mais próximo a abordagem endpoint (TACHARD, 2007).

A sua versão 2003 trouxe melhorias para as categorias de impactos utilizadas tornando modelos de caracterização mais regionalizados, com foco nas condições Europeias. Esta versão menos global não contempla a classificação dos resíduos sólidos gerados e alterou os fatores de caracterização para as categorias de impactos não globais (acidificação, eutrofização, formação de ozônio fotoquímico, ecotoxicidade e toxicidade humana) aumentando a relevância da caracterização na versão EDIP03. As referências para a normalização também foram alteradas neste método. (HAUSCHILD; POTTING, 2005; SILVA, 2012).

O EDIP 2003 possui uma abordagem típica midpoint, abrangendo a maioria dos impactos relacionados a emissões, uso de recursos e impactos no meio ambiente de trabalho. As categorias de impacto abordadas são: acidificação, eutrofização terrestre, exposição fotoquímica do ozônio em plantas e seres humanos, eutrofização aquática, toxicidade humana e ecotoxicidade (HAUSCHILD; POTTING, 2005).

O guia metodológico do EDIP 2003 (HAUSCHILD; POTTING, 2005) recomenda que o mesmo seja usado como uma alternativa para ao EDIP97 em uma caracterização local genérica (de preferência europeia). O EDIP97 ainda pode ser utilizado se um novo estudo ACV puder ser comparado com um estudo anterior que se baseou na metodologia e nos fatores do EDIP97.

\subsection{Impact 2002+}

O método Impact 2002+ é uma metodologia de avaliação de impactos originalmente desenvolvida no Instituto Federal de Tecnologia da Suíça (EPFL), em Lausanne. A metodologia propõe uma implementação viável da combinação de métodos de avaliação de impactos com abordagem orientada ao problema (midpoint) e a orientada ao dano (endpoint) (ECOINVENT, 
2010).

A proposta do método Impact2002+ é a junção dos métodos midpoint com endpoint, a fim de absorver suas respectivas limitações e agrupar os pontos positivos dos métodos mais utilizados mundialmente em estudos de ACV. Goedkoop et al (2008) afirmam que o Impact 2002+ é, em termos gerais, a combinação entre o Impact 2002 (em sua primeira versão) e os métodos EcoIndicator99, CML 2000 e IPCC.

A Figura 3 ilustra as categorias de impactos médias (midpoint) e de danos consideradas (endpoint) no método Impact2002+.

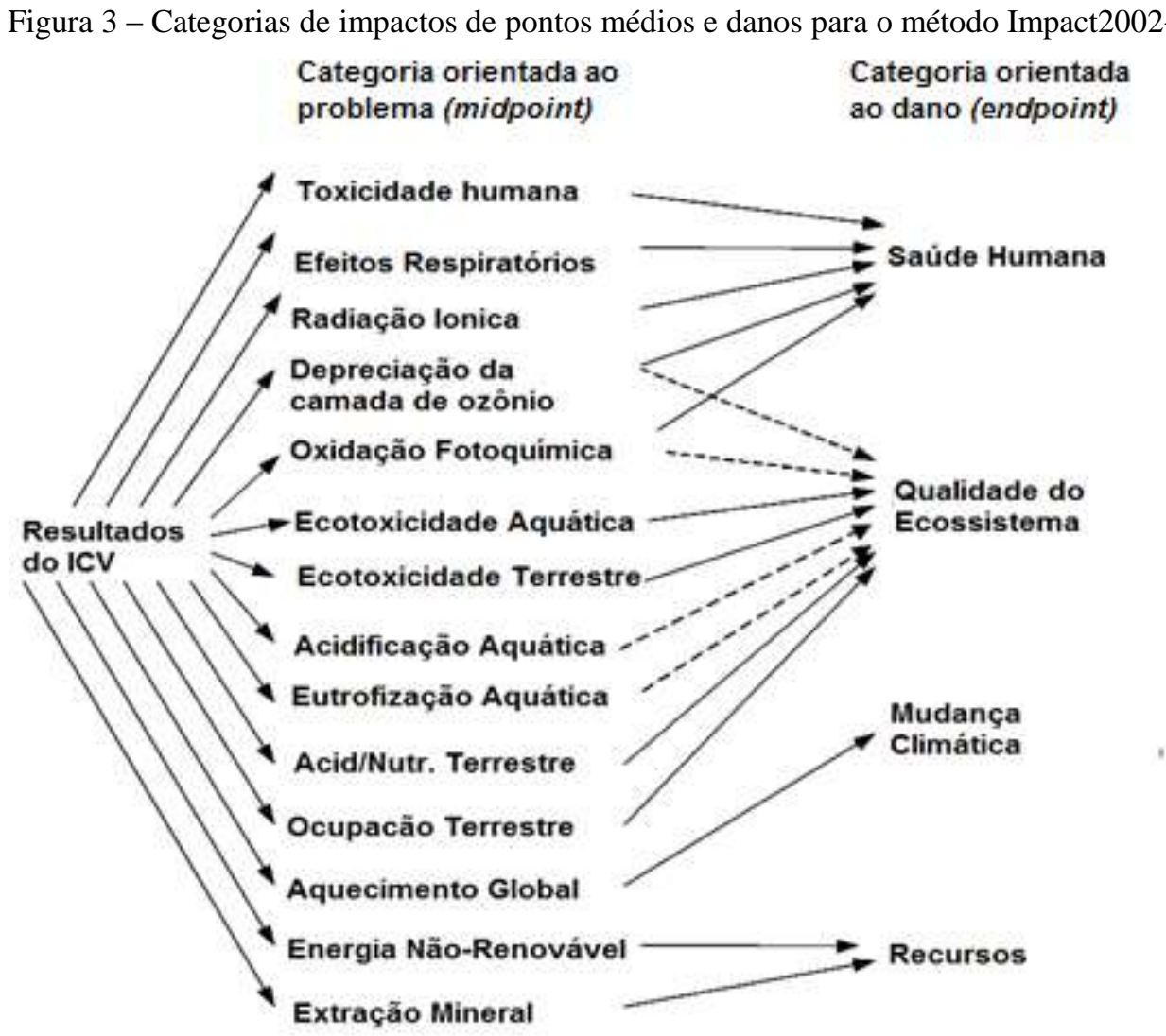

Fonte: Ecoinvent (2010b)

Todos os resultados do ICV são ligados às quatorze (14) categorias de ponto médio, e estas por sua vez, ligadas as quatro (4) categorias de danos. A publicação original deste método (JOLLIET et al, 2003) pode ser útil para a compreensão da metodologia de impacto, para os fatores para as caracterizações dos fluxos, e para os procedimentos de cálculos envolvidos neste método.

\section{5 $\quad \operatorname{ReCiPe} 2008$}

ReCiPe 2008 é um método de AICV que leva em seu nome o acrônimo representado pelas iniciais das organizações responsáveis pelas principais contribuições em seu desenvolvimento: 
RIVM, a Radboud University Nijmegen, o centro de pesquisa CML da Universidade de Leiden e a empresa PRé Consults.

O ReCiPe é um método de caráter combinativo entre abordagens midpoint e endpoint para avaliação de impactos do ciclo de vida. A sua metodologia é harmonizada em termos de princípios de modelagem e escolhas, oferecendo resultados orientados a problemas e a danos (GOEDKOOP et al, 2009).

Este método foi desenvolvido utilizando como base os métodos CML e Eco-Indicator 99. O ReCiPe 2008, seguindo características do Eco-Indicator 99, foi desenvolvido em três versões de ponderação e normalização das categorias de impacto, baseadas na teoria de perspectivas culturais (desenvolvida por Mary Douglas e Aaron Wildavsky), onde estabelece-se conjuntos consistentes de escolhas subjetivas que são agrupados em três perspectivas: Individualista, Hierarquizada e Igualitária (ALVARENGA, 2010).

De acordo com Goedkoop et al (2009), as categorias de impacto de ambiental compreendidas no método ReCiPe são:

a) Midpoint: Acidificação terrestre, Depleção da camada de ozônio, Depleção de água, Depleção de combustíveis fósseis, Depleção de recursos minerais, Ecotoxicidade em água doce, Ecotoxicidade marinha, Ecotoxicidade terrestre, Eutrofização de água doce, Eutrofização marinha, Formação de material particulado, Formação de oxidantes fotoquímicos, Mudanças climáticas, Ocupação de solo agrícola, Ocupação de solo urbano, Radiação ionizante, Toxicidade humana e Transformação de solo natural.

b) Endpoint: Danos à saúde humana, danos à diversidade dos ecossistemas, e danos à disponibilidade de recursos.

No nível de midpoint, as categorias de impactos são relatadas em função de unidades individuais para cada fator. Enquanto no nível endpoint, é implementada uma pontuação geral única. Esta pontuação não se refere apenas as três categorias de danos (saúde humana, ecossistemas e recursos), mas também as contribuições de vários indicadores midpoint (que possuem participação respectiva na categoria de danos) (GOEDKOOP et al, 2009).

\section{Adoção de múltiplos métodos para especificidades brasileiras}

A inexistência de um método de avaliação de impactos do ciclo de vida específico para as condições brasileiras implica em dificuldades no momento de seleção de métodos para um estudo ACV.

Cada ACV possui uma particularidade, um objetivo e um escopo diferente, portanto, na escolha dos métodos de AICV devem-se levar em conta as características e os objetivos pretendidos 
com o estudo. Um levantamento do estado da arte de estudos de ACV já desenvolvidos em características similares auxilia a seleção dos métodos a serem adotados.

Alvarenga (2010) afirma que devido à dificuldade de encontrar um método de AICV ideal para um determinado estudo, já que todos apresentam vantagens e desvantagens, uma alternativa plausível é realizar o estudo ACV com mais de um método de AICV. Este fato dá suporte à tomada de decisão mais confiável. Quando ocorre uma convergência dos resultados obtidos, a adoção de métodos múltiplos fornece maior confiança ao decisor. Em contrapartida, quando há divergência, o decisor pode definir critérios de escolha do método que aceitará como resposta final (maior diferença nos resultados, afinidade das categorias de impacto, etc.) ou escolher um novo critério de decisão.

Quanto às incertezas nos resultados, a adoção de mais de um método de AICV figura-se como uma alternativa interessante para a análise de resultados de estudos ACV nas especificidades brasileiras. De acordo com Silva (2012), adotando mais de um método, é possível a comparação dos resultados do estudo, e verificar se as conclusões e recomendações tomadas são influenciadas conforme os métodos escolhidos.

O Quadro 2 apresenta alguns exemplos de estudos de ACV brasileiros que aplicaram múltiplos métodos para AICV.

Quadro 1 - Métodos para AICV

\begin{tabular}{|l|l|l|}
\hline \multicolumn{1}{|c|}{ Título } & \multicolumn{1}{|c|}{ Métodos Empregados } & \multicolumn{1}{c|}{ Referência } \\
\hline $\begin{array}{l}\text { Avaliação de métodos de AICV: Um estudo de caso } \\
\text { de quatro cenários de ração para frangos de corte }\end{array}$ & $\begin{array}{l}\text { CML, Eco-Indicator99, Pegada } \\
\text { Ecológica, Pegada de Carbono }\end{array}$ & Alvarenga (2010) \\
\hline $\begin{array}{l}\text { Avaliação do ciclo de vida da produção do painel de } \\
\text { madeira mdp no Brasil }\end{array}$ & CML, EDIP, USEtox & Silva (2012) \\
\hline $\begin{array}{l}\text { Comparison of the ecological footprint and a life } \\
\text { cycle impact assessment method for a case study on } \\
\text { Brazilian broiler feed production }\end{array}$ & CML, Pegada Ecológica & $\begin{array}{l}\text { Alvarenga et al } \\
(2012)\end{array}$ \\
\hline $\begin{array}{l}\text { Comparative LCA of ethanol versus gasoline in } \\
\text { Brazil using different LCIA methods }\end{array}$ & $\begin{array}{l}\text { CML, Impact 2002+, EDIP2003, } \\
\text { Eco-Indicator99,TRACI 2, ReCiPe, } \\
\text { Ecological Scarcity 2006 }\end{array}$ & $\begin{array}{l}\text { Cavalett et al } \\
(2012)\end{array}$ \\
\hline
\end{tabular}

Fonte: Autoria própria (2012)

Em análise dos estudos apresentados, nota-se que a possibilidade de comparação dos resultados gerados por métodos diferentes apresenta-se como uma característica predominante. De acordo com Alvarenga (2010), para a adoção do método, é altamente recomendável que se conheça a fundo um método antes de utilizá-lo, inicialmente para selecionar aquele que atenda as necessidades do estudo, mas para que também se possa justificar discrepâncias nos resultados e estimar impactos negligenciados.

Por sua vez, a peculiaridade de cada estudo e o estado da arte do produto ou processo que está sendo estudado são direcionadores da seleção. O tipo de abordagem de impacto (midpoint ou endpoint) que um estudo ACV irá requerer também atua como facilitador na seleção de método de AICV. Se utilizados para caráter comparativo, os métodos devem possuir categorias de impactos 
similares, em caso contrário (quando o estudo deseja revelar impactos orientados ao problema e ao dano), o estudo recorrerá a utilização de métodos com categorias de impactos diferentes, de midpoints e endpoints.

\section{Considerações Finais}

A ACV é uma ferramenta de gestão ambiental que pode ser amplamente utilizada para avaliar os impactos causados por produtos, processos e serviços sobre o meio ambiente durante todo o seu ciclo de vida. A ferramenta vem sendo amplamente aplicada na comunidade científica, em indústrias, e organizações que pretendem avaliar o impacto de suas atividades na perspectiva de ciclo de vida.

A escolha do método de AICV que será utilizado é um ponto crucial para que os resultados obtidos apresentem maior confiabilidade. Como o Brasil não apresenta um método de avaliação de impactos consolidado condizente com as especificidades brasileiras, as incertezas geradas no estudo podem ser reduzidas adotando-se múltiplos métodos de avaliação, o que garantirá maior confiabilidade aos resultados obtidos.

Para a escolha do método é importante ressaltar que o mesmo deve estar condizente com os objetivos traçados e os resultados esperados da ACV. Qualquer que seja o(s) método(s) escolhido é de fundamental importância que haja um estudo aprofundado sobre suas características e aplicabilidade. Deste modo, um levantamento bibliográfico de ACV's de produtos, processos ou serviços similares ao do estudo que será desenvolvido, verificando quais são os métodos que vem sendo aplicados, pode auxiliar e justificar o processo de seleção de métodos para a ACV.

\section{Abstract}

The Life Cycle Impact Assessment (LCIA) is done through a combination of life cycle inventory (LCI) data and specifics impact categories. This process can receive assistance of different LCIA methods. However, there are no methods for the Brazilian specifics and it is one of the difficulties in LCA methodology in Brazil. Thus, this study aims to discuss the adoption of multiple methods for LCIA in Brazilian specifications. For this, was developed an exploratory research. Concepts regarding LCA, LCIA methods and the adoption of multiple methods for Brazilian specificities were discussed. Was noted that comparison of LCA`s results by the use of different methods are presented as a crucial feature. Thus the uncertainties in results of Brazilian LCA`s studies can be reduced by adopting multiple methods.

Key-words: Life Cycle Assessment (LCA), Life Cycle Impact Assessment (LCIA), LCIA methods.

\section{Referências}

ALVARENGA, R. A. F. Avaliação de métodos de AICV: Um estudo de caso de quatro cenários de ração para frangos de corte. 2010. 158 f. Dissertação (Mestrado) - Curso de Engenharia Ambiental, Departamento de Programa de Pósgraduação em Engenharia Ambiental, Universidade Federal de Santa Catarina, Florianópolis, 2010. 
ALVARENGA, R. A. F.; SILVA JÚNIOR, V. P.; SOARES, S. R. Comparison of the ecological footprint and a life cycle impact assessment method for a case study on Brazilian broiler feed production. Journal of Cleaner Production, v. 28, p. 25-32, 2012.

cross ref

ASSOCIAÇÃO BRASILEIRA DE NORMAS TÉCNICAS (ABNT). NBR ISO 14040: Gestão Ambiental - Avaliação do ciclo de vida - Princípios e estrutura. Brasil, 2009a.

ASSOCIAÇÃO BRASILEIRA DE NORMAS TÉCNICAS (ABNT). NBR ISO 14044: Gestão Ambiental - Avaliação do ciclo de vida - Requisitos e Orientações. Brasil, $2009 \mathrm{~b}$.

BARE J.C., NORRIS, G.A., PENNINGTON D.W., MCKONE T. TRACI, The Tool for the Reduction and Assessment of Chemical and Other Environmental Impacts. Journal of Industrial Ecology, v. 6, n. 3-4, 2003.

BARE J.C, HOFSTETTER P., PENNINGTON D.W, DE HAES HA U. (2000) Life cycle impact assessment midpoints vs. endpoints: the sacrifices and the benefits. International Journal of Life Cycle Assessment , v.5, n.5, 2000.

BRAND G., BRAUNSCHWEIG A., SCHEIDEGGER A., SCHWANK O. Bewertung in Oekobilanzen mit der Methode der ökologischen Knappheit Oekofaktoren 1997. BUWAL Schriftenreihe Umwelt Nr. 297. BUWAL, Bern, 1998.

CAVAlETT, O.; CHAGASM, M. F.; SEABRA, J. E. A.; BONOMI, A. Comparative LCA of ethanol versus gasoline in Brazil using different LCIA methods. International Journal of Life Cycle Assessment, 2012 [in press].

cross ref

CCI . ILCD Handbook: analysis of existing environmental impact assessment methodologies for use in life cycle assessment, 2010. Disponível em: <http://lct.jrc.ec.europa.eu/pdf-directory/ILCD-Handbook-LCIA-Backgroundanalysis-online-12March2010.pdf>. Acesso em: 13 fev. 2012.

CHAUHAN, M. K; CHAUDHARY, V. S.; SAMAR, S. K. Life cycle assessment of sugar industry: A review. Renewable and Sustainable Energy Reviews, v.15. n.7, p. 3445-3453, abr. 2011.

cross'ref

ECOINVENT. Ecoinvent data Vol.2.2: Implementation of Life Cycle Impact Assessment Method; Suíça: Swiss Centre for Life Cycle Inventories, 2010.

FRISCHKNECHT R., STEINER R., JUNGBLUTH N. Methode der ökologischen Knappheit - Ökofaktoren 2006. Methode für die Wirkungsabschätzung in Ökobilanzen. Umwelt-Wissen Nr. 0906, Bundesamt für Umwelt, Bern, 2009.

GARRAÍN, D.; VIDAL, R.; MARTÍNEZ, P.; MUÑOZ, C. Análisis del Ciclo de Vida de los Procesos de Recubrimiento Metálico de Termoplásticos. Información Tecnológica, v. 2, n.2, p. 59-64, 2010.

GOEDKOOP M.; HEIJUNGS R.; DE SCHRYVER A.; STRUIJS J.; VAN ZELM R. ReCiPe 2008: A life cycle impact assessment method which comprises harmonized category indicators at the midpoint and the endpoint level / Report I: Characterization. Holanda: Ministerie van VROM, Den Haag, 2009.

GOEDKOOP M.; HOFSTETTER P.; MÜLLER-WENK R.; SPRIENSMA R. The Eco-Indicator 98 Explained. The International Journal of Life Cycle Assessment, v.3, n. 6, p.352-360, 1998.

cross ref

GOEDKOOP M.; SPRIENSMA R. Methodology Annex: The Eco-indicator 99 - A damage oriented method for life cycle impact assessment. Holanda: PRé Consultants, 2000b.

GOEDKOOP, M.; OELE, M.; SCHRYVER, A.; VIEIRA, M. SimaPro Database Manual: Methods Library. Holanda: PRé Consultants, 2008. Disponível em: <www.pre.nl>. Acesso em: Fevereiro 2012.

GOEDKOOP, M.; SPRIENSMA, R. The Eco-indicator 99: A damage-oriented method for life cycle impact assessment. Methodology report, second edition. Holanda: Pré Consultants, B.V.Amersfoort, 2000a. 
GUINÈE J. B. et al Life cycle assessment: An operational guide to the ISO standards - Characterisation and Normalisation Factors. Holanda: Ministry of Housing, Spatial Planning and Environment (VROM) and Centre of Environmental Science (CML), 2001a.

GUINÈE J. B. et al Life cycle assessment: An operational guide to the ISO standards - Part 3: Scientific Background. Holanda: Ministry of Housing, Spatial Planning and Environment (VROM) and Centre of Environmental Science (CML), 2001b.

GUINÈE J. B. et al Life cycle assessment: An operational guide to the ISO standards - Parts 1 and 2. Holanda: Ministry of Housing, Spatial Planning and Environment (VROM) and Centre of Environmental Science (CML), 2001c.

GUINÉE, J. B. et al Life Cycle Assessment: past, present, and future. Environmental Science and Technology, v. 45, n.1, p.90-96, 2011.

cross ref

HAUSCHILD, M. Z. et al Identifying best existing practice for characterization modeling in life cycle impact assessment. International Journal of Life Cycle Assessment, set. 2012.

\section{cross ref}

HAUSCHILD, M. Z.; POTTING, J. Spatial differentiation in life cycle impact assessment - the EDIP2003 methodology. Guidelines from the danish environmental protection agency. Environmental News, n. 80, Copenhagen, Dinamarca, 2005.

HAUSCHILD, M. et al Building a Consensus Model for Life Cycle Impact Assessment of Chemicals: the Search for Harmony and Parsimony. Environmental Science Technololy, v.42, n.19, 2008.

HAYASHI, K., NAKAGAWA, A., ITSUBO, N., INABA, A. Expanded Damage Function of Stratospheric Ozone Depletion to Cover Major Endpoints Regarding Life Cycle Impact Assessment. International Journal of Life Cycle Assessment, v.11, n.3, 2006.

cross ref

HUIJBREGTS, M. A. J., HELLWEG S., FRISCHKNECHT R., HUNGERBÜHLER K.; HENDRIKS A. J. Ecological Footprint Accounting in the Life Cycle Assessment of Products. Ecological Economics, v.64, n.4, 2006.

ITSUBO, N., SAKAGAMI, M., WASHIDA, T., KOKUBU, K.; INABA, A. Weighting Across Safeguard Subjects for LCIA through the Application of Conjoint Analysis. International Journal of Life Cycle Assessment, v.9, n.3, 2004. cross ref

JÚNIOR, V. A.; DEMAJOROVIC, Jacques. Modelos e ferramentas de gestão ambiental: desafios e perspectivas para organizações. São Paulo. Ed. Senac, 2010.

JIJAKLI, K. et al How green solar desalination really is? Environmental assessment using life-cycle analysis (LCA) approach. Desalination, v. 287, n.15, p. 123-131, fev. 2012.

\section{cross ref}

JOLLIET, O. et al IMPACT 2002+: A New Life Cycle Impact Assessment Methodology. International Journal of Life Cycle Assessment, v.8, n.6, p.324-330, 2003.

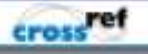

KEMNA, R., VAN ELBURG, M., LI, W., VAN HOLSTEIN, R. MEEuP - The methodology Report. EC, Brussels, 2005.

LOFGREN, B.; TILLMAN, A. M.; RINDE, B. Manufacturing actor's LCA. Journal of Cleaner Production, v. 19, p. 2025-2033, 2011.

cross ref

LUZ, L. M. Proposta de modelo para avaliar a contribuição dos indicadores obtidos na análise do ciclo de vida sobre a geração de inovação na indústria. 2011. 165 f. Dissertação (Mestrado em Engenharia de Produção) - 
Programa de Pós-Graduação em Engenharia de Produção, Universidade Tecnológica Federal do Paraná. Ponta Grossa, 2011.

MYLLYVIITA, T.; HOLMA, A.; ANTIKAINEN, R.; LÄHTINEN, K.; LESKINEN, P. Assessing environmental impacts of biomass production chains: application $\mathrm{f}$ life cycle assessment (LCA) and multi-criteria decision analysis (MCDA). Journal of Cleaner Production, v. 29-30, p.238-245, 2012.

cross ref

OLSEN, S. I.; CHRISTENSEN, F. M.; HAUSCHILD, M.; PEDERSEN, F.; LARSEN, H. F.; TORSLOV, J. Life cycle impact assessment and risk assessment of chemicals - a methodological comparison. Environmental Impact Assessment Review. v.21, n.4, p. 385-404, 2001.

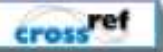

PIERAGOSTINI, C.; MUSSAT M. C. I.; AGUIRRE, P. On process optimization considering LCA methodology. Journal of Environmental Management, v.96, p. 46-54, 2012.

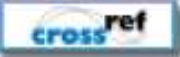

PIZZOL, M.; CHRISTENSEN, P.; SCHMIDT, J. H; THOMSEN, M. Eco-toxicological impact of "metals" on the aquatic and terrestrial ecosystem: a comparison between eight different methodologies for life cycle impact assessment (LCIA). Journal of Cleaner Production, v. 19, p.687-698, 2011a.

cross ref

PIZZOL, M.; CHRISTENSEN, P.; SCHMIDT, J. H; THOMSEN, M. Impacts of "metals" on human health: a comparison between nine different methodologies for life cycle impact assessment (LCIA). Journal of Cleaner Production, v. 19, p.646-656, 2011 b.

cross ref

RESTREPO, Á.; MIYAKE, R.; KLEVESTON, F.; BAZZO, E. Exergetic and environmental analysis of a pulverized coal power plant. Energy, v. 45, 2012.

ROSENBAUM R. K., et al USEtox - the UNEP-SETAC toxicity model: recommended characterisation factors for human toxicity and freshwater ecotoxicity in life cycle impact assessment. International Journal of Life Cycle Assessment, v.13, 2008.

SANTOS, M. F. N. Análise dos Impactos na Construção Civil: Avaliação do Ciclo de Vida em Chapas de Partículas para forros. 151f. Dissertação (Mestrado) - Faculdade de Engenharia de Bauru, Universidade Estadual Paulista, Bauru, 2010.

SHERWANIA, A. F.; USMANIB, J. A.; VARUNC. Life cycle assessment of solar PV based electricity generation systems: A review. Renewable and Sustainable Energy Reviews, v. 14, n.1, p. 540-544, 2010.

cross ${ }^{\text {ref }}$

SILVA, D. A. L. Avaliação do ciclo de vida da produção do painel de madeira MDP no Brasil. 2012. 207 f.

Dissertação (Mestrado) - Curso de Ciência e Engenharia de Materiais, Departamento de Programa de Pós-graduação

SILVA, R. B; KAZIMI, M. S.; HEJZLAR, P. Nuclear fuel recycling: National and regional options for the US nuclear energy system. Energy \& Environmental Science, v.3, 2010.

SOUZA, D. P.; MENDONÇA, F. M.; NUNES, K. R. A.; VALLE, R. Environmental and Socioeconomic Analysis of Producing Biodiesel from Used Cooking Oil in Rio de Janeiro: The Case of the Copacabana District. Journal of Industrial Ecology, v. 16, n. 4, p. 655-664, ago. 2012.

STEEN B. A systematic approach to environmental priority strategies in product development (EPS). Version $2000-$ Models and data of the default method. CPM Report 1999b, Technical Environmental Planning, Chalmers University of Technology, Sweden. 1999b.

STEEN B. A systematic Approach to Environmental Priority Strategies in Product Development (EPS). Version 2000 General system characteristics. CPM Report 1999:4, Chalmers University of Technology, Gothenburg, Sweden, 1999a. 
STEEN B. Identification of significant environmental aspects and their indicators. NORDEPE, CPM Report Nr 2001:7. Chalmers University of Technology, Gothenburg, Sweden, 2001.

SUER, P.; PALEDAL, N. S.; NORRMAN, J. LCA for site remediation: a literature review. Soil and Sediment Contamination, v. 13, p. 415-425, 2004.

cross ref

SUH, S.; HUPPES, G. Methods for Life Cycle Inventory of a product. Journal of Cleaner Production, v. 13, n.7, p. 687-697, jun. 2005.

cross ref

TACHARD, A. L. R. S. Desenvolvimento de fatores de normalização de impactos ambientais regionais para Avaliação do Ciclo de Vida (ACV) de produtos no estado de São Paulo. 2007. Tese (Doutorado em Ciência da Engenharia Ambiental) -Programa de Pós Graduação em Ciências da Engenharia Ambiental, Departamento de Hidráulica e Saneamento, Escola de Engenharia de São Carlos, Universidade de São Paulo, São Paulo, 2007.

TAKEDA, Adriane. Levantamento de métodos de avaliação de impacto de ciclo de vida (AICV) e análise comparativa dos métodos mais utilizados. 2008. 129 f. Monografia (Graduação) - Curso de Engenharia Ambiental, Departamento de Escola de Engenharia, Universidade de São Paulo, São Carlos, 2008.

BULLE, C. GODIN, J. REID, C. DESCHÊNES, L.Deschênes. LUCAS - A New LCIA Method Used for a CAnadian-Specific Context. International Journal of Life Cycle Assessment, v.12, n.2, 2007.

Wackernagel, M., Monfreda, C., Moran, D., Wermer, P., Goldfinger, S., Deumling, D., Murray, M. —National Footprint and Biocapacity Accounts 2005: The underlying calculation methodll. Global Footprint Network, Oakland, California, USA, 2005.

ZHOU, J.; CHANG, V. W.C.; FANE, A. G. Environmental life cycle assessment of reverse osmosis desalination: The influence of different lifecycle impact assessment methods on the characterization results. Desalination, v. 283, n.1, p. 227-236, 2011.

crossef

\section{Agradecimentos}

Os autores agradecem a CAPES (Coordenação de Aperfeiçoamento de Pessoal de Nível Superior) pelo apoio recebido para o desenvolvimento da pesquisa.

\section{Dados dos Autores:}

Nome completo: Cassiano Moro Piekarski

Filiação institucional: Universidade Tecnológica Federal do Paraná - Campus Ponta Grossa

Departamento: Programa de Pós-Graduação em Engenharia de Produção

Função ou cargo ocupado: Mestrando em Engenharia de Produção

Endereço completo para correspondência: Av. Monteiro Lobato, s/n. Km.4

Telefones para contato: (42) 3220-4876

e-mail: cassianopiekarski@gmail.com

Nome completo: Leila Mendes da Luz

Filiação institucional: Universidade Tecnológica Federal do Paraná - Campus Ponta Grossa

Departamento: Programa de Pós-Graduação em Engenharia de Produção

Função ou cargo ocupado: Doutoranda em Engenharia de Produção 
Endereço completo para correspondência: Av. Monteiro Lobato, s/n. Km.4

Telefones para contato: (42) 3220-4876

e-mail: leila.mendesdaluz@gmail.com

Nome completo: Lidiana Zocche

Filiação institucional: Universidade Tecnológica Federal do Paraná - Campus Ponta Grossa

Departamento: Programa de Pós-Graduação em Engenharia de Produção

Função ou cargo ocupado: Mestranda em Engenharia de Produção

Endereço completo para correspondência: Av. Monteiro Lobato, s/n. Km.4

Telefones para contato: (42) 3220-4876

e-mail: lidianazocche@hotmail.com

Nome completo: Antonio Carlos de Francisco

Filiação institucional: Universidade Tecnológica Federal do Paraná - Campus Ponta Grossa

Departamento: Programa de Pós-Graduação em Engenharia de Produção

Função ou cargo ocupado: Professor

Endereço completo para correspondência: Av. Monteiro Lobato, s/n. Km.4

Telefones para contato: (42) 3220-4876

e-mail: acfrancisco@utfpr.edu.br

Enviado em: 20/03/2012

Aprovado em: 27/09/2012 